\title{
Une maladie de la vision chez une porteuse d'une isodisomie uniparentale du chromosome 14
}

Les mécanismes de la vision des couleurs sont bien établis. On sait $(\mathrm{m} / \mathrm{s}$ $n^{\circ} 7$, vol. 2, p. 402) que les gènes des pigments vert et rouge sont portés par le chromosome $\mathrm{X}$ sur son bras long, leurs défauts donnant naissance aux diverses variétés du daltonisme, liécs au sexc. Lc gène du pigment bleu sic̀ge sur le bras long du chromosome 7 ; le déficit correspondant, récessif autosomique, est la très rare tritanopsie. Mais il existe une autre affection, dans laquelle la vision des couleurs est totalement abolic du fait du non-développement des photorécepteurs des cônes. C'est l'achromatopsic congénitalc complète, appcléc aussi cécité aux coulcurs, ou cécité diurne, car la vision est meillcure la nuit ; s'y ajoutent une réduction de l'acuité visuelle centralc, unc réaction paradoxale de la pupille, en principe sans manifestations extra-oculaires. Le gène de cette maladic récessive autosomique n'a pas été encore localisé. Une équipe de Houston (TX, USA) a eu l'occasion d'examiner une femme de 20 ans attcinte de cette affection, qui présentait en outre unc série de symptômes : petite taille $(1,44 \mathrm{~m})$, pubcrté précoce, retard intellectuel modéré, avortements à répétitions. Cet ensemble incita à entreprendre une étude cytogénétique. Le caryotype révéla une formule 45 , $\mathrm{XX}, \operatorname{rob}(14 ; 14)$ ce qui signifie que les deux chromosomes 14 étaient unis par une translocation robertsonienne (fusion de deux chromosomes par leurs extrémités). Le caryotype de la mère ct de deux autres enfants était normal; le père était décédé.

On entreprit ensuite unc étude moléculaire afin de découvrir l'origine des 14 fusionnés. On utilisa pour cela onze marqueurs couvrant l'enscmble du 14. La comparaison de la mère et de ses trois enfants permit en outre de reconstituer l'haplotype du père. La conclusion fut formclle : les chromosomes 14 de la fille ne contenaient aucune contribution paternelle; ils toutes les sondes employécs, et dérivaient donc d'un même chromosome maternel dupliqué. De plus, la malade possédait une dose double de l'allèle unique à chaque locus. Des analyses semblables portant sur d'autres chromosomes ont montré une contribution normale des deux parents, ainsi que pour l'ensemble du génome des deux autres enfants, incluant les chromosomes 14. Cette translocation robertsonienne fournit donc un chromosome effectivement dérivé d'un seul 14 maternel, et révèle une isodisomic d'origine maternclle. Rappelons (voir $\mathrm{m} / \mathrm{s} n^{\circ} 1$, vol. 6, p. 57) qu'on appelle disomic uniparentale le fait de recevoir dcux autosomes homologues d'un même parent ; il s'agit d'une hétérodisomic si les deux chromosomes sont différents et d'une isodisomie si c'est le même qui a subi une duplication. Le premier exemple a été fourni par deux cas de mucoviscidose, liés à une isodisomic du chromosome 7 d'origine maternelle ; la mère était hétérozygote et le père normal ; le risque évident de l'isodisomie est en effet que la réduction à l'état homozygote peut révéler une maladie récessive, alors même qu'un seul parent est hétérozygote.

C'est bien de cette façon que doit être interprété ce cas d'achromatopsie. Le mécanisme le plus probable est que c'est une homozygotie pour le locus de la maladie qui a été provoquéc par l'isodisomic. On doit donc conclure que le locus, encore inconnu, de cette affection, se trouve sur le chromosome 14. Mais ce cas pose plusicurs questions supplémentaires : il n'cxiste pas habituellement de symptômes extra-oculaires dans l'achromatopsic. A quoi sont-ils dus dans le cas présent ? A une coïncidence, à la révélation fortuitc d'autres anomalics récessives, ou à une empreinte génomique, du fait que les gènes hérités des deux parents ne seraient pas équivalents? on se rappelle, par exemple, que les deux enfants atteints de mucoviscidose par suite d'isodisomie avaient une taille réduite. Un argument en favcur du rôle de la disomie uniparentale est tiré d'unc observation voisine [2] : un garçon, de petite taille, et ayant manifesté unc puberté précoce, avait hérité de sa mère unc isodisomic de ses chromosomes 14 , à la suite d'une translocation robertsonienne entre un 13 et un 14 . Les travaux à venir devraient se consacrer à confirmer la localisation sur le 14 par d'autres méthodes. Les auteurs [1] signalent que l'on connaît, sur ce chromosome, deux gènes spécifiques de la rétine: $N R S-1$, dont le polypeptide dérivé possède une fermeture à glissière de leucine (leucine zipper) [3], et HOX10 (RET1), gène à homéoboîte contrôlé au cours du développement.

On peut cnfin faire deux remarques générales : lorsque, au cours de translocations équilibrécs, notamment robertsoniennes, on trouve les symptômes d'une maladic récessive autosomique, ou un syndrome complexe, il faut rechercher une disomie uniparentale ; d'autre part, ce type d'observation devrait inciter à reprendre - à la recherche de syndromes passés inaperçus - les cas de translocation robertsonienne parus dans la littérature ; et ce, bien entendu, pas seulement pour le chromosome 14.

J.C.D.

1. Pentao L, Lewis RA, Ledbetter DH, Patel PI, Lupski JR. Maternal uniparental isodisomy of chromosome 14: association with autosomal recessive rod monochromacy. Am J Hum Genet $1992 ; 50: 690-9$

2. Temple IK, Cockwell A, Hassold T, Pettay D, Jacobs P. Maternal uniparental disomy for chromosome 14. J Med Genet 1991; 28 : 511-4. 3. Jackson A, Pawar H, Xu J, Swaroop A Molecular structure, chromosomal localization and conservation of NRS-1, a novel human neural retina specific gene of jun/fos oncogene family. Am J Hum Genet 1991 ; 49 : A2308.

4. De Chen J, Ploder L, Collins L, et al. Chromosomal sublocalization and cellular expression of the retinal homeobox gene HOX10. $A m J$ Hum Genet 1990) ; 47 (suppl.) : A102. 